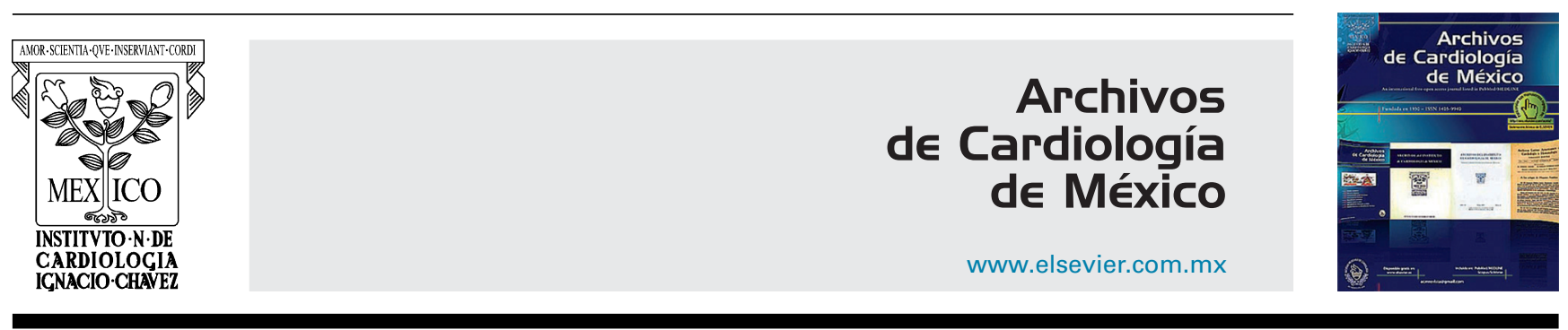

IMAGEN EN CARDIOLOGÍA

\title{
Estudio histopatológico de arteria coronaria, tratada con stent metálico, posterior a la técnica de electrodeposición
}

\section{Histopatological study of stented coronary artery treated with the electrodeposition technique}

\author{
Mario Zúñiga-Ayala ${ }^{\mathrm{a}, *}$, Octavio Juárez-Alvarado ${ }^{\mathrm{a}}$, Alberto Aranda-Fraustro ${ }^{\mathrm{b}}$, \\ Rodrigo Velazquéz-Espejel ${ }^{b}$, Brayans Becerra-Luna ${ }^{c}$ y Marco Peña-Duque ${ }^{a}$ \\ a Unidad de Investigación, Departamento de Hemodinámica, Instituto Nacional de Cardiología Ignacio Chávez, Ciudad \\ de México, México \\ b Departamento de Anatomía Patológica, Instituto Nacional de Cardiología Ignacio Chávez, Ciudad de México, México \\ c Departamento de Instrumentación Electromecánica, Instituto Nacional de Cardiología Ignacio Chávez, Ciudad de México, \\ México
}

Recibido el 23 de mayo de 2016; aceptado el 12 de septiembre de 2016

El estudio histopatológico de arterias coronarias con stent resulta un proceso complicado bajo las técnicas descritas en la actualidad ${ }^{1}$, ya que utilizan la inclusión en resina de metacrilato de metilo (MMA) para su posterior procesamiento por equipos complejos, como el uso de cuchillas de tungsteno para corte fino, y otros como el corte con sierra rápida de punta de diamante. El procedimiento y el uso de los equipos antes mencionados requieren, en gran medida, de la habilidad del operador, pues su uso no

\footnotetext{
* Autor para correspondencia. Unidad de Investigación de Hemodinámica, Instituto Nacional de Cardiología, Juan Badiano N. ${ }^{\circ}$, Col. Sección XVI, Delegación Tlalpan CP 14080; Ciudad de México, México; Teléfono: +55 55732911, Extensión: 1467.

Correo electrónico: zuniga_mario@live.com (M. Zúñiga-Ayala).
}

es trivial, además del alto recurso económico necesario. La electrodeposición consiste en la disolución electroquímica de metales a través de un líquido conductor o electrolito (solución ácida), donde un electrodo (metal con carga positiva) se oxida para donar sus electrones a otro electrodo (metal con carga negativa) reduciendo este, el fenómeno es denominado electrólisis. En este contexto, la técnica ha demostrado ser objetivada en el estudio de arterias tratadas con stents metálicos ${ }^{2}$, pues permite disolver estos.

A continuación presentamos distintos cortes histológicos (figs. 1-3) de una arteria coronaria descendente anterior con previa colocación de stent metálico tras la aplicación de electrodeposición, objetivando la reproducibilidad de la técnica y su valiosa aportación al estudio histopatológico de la reacción celular al stent. 


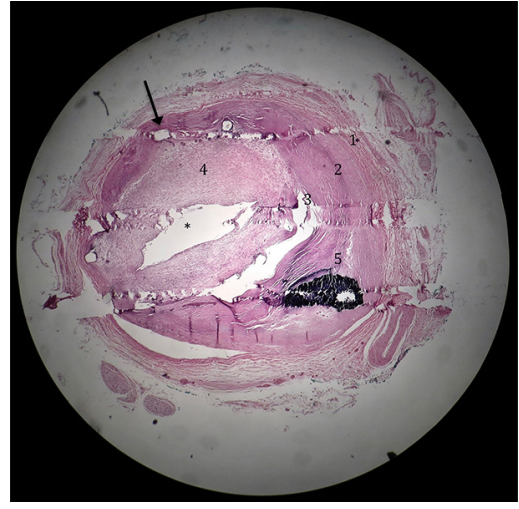

Figura 1 Fotomicrografía de arteria coronaria posterior a la electrodeposición. Se observa el espacio previamente ocupado por strut de stent (flecha), las 3 capas de la arteria de afuera hacia adentro, túnica adventicia (1), túnica media (2), túnica íntima con placa fibroesclerótica (3), proliferación extensa de capa neointimal (4) que ocluye la luz del vaso $\left({ }^{*}\right)$. Calcificación de placa esclerótica (5) $(\mathrm{H \& E} \times 2.5)$.

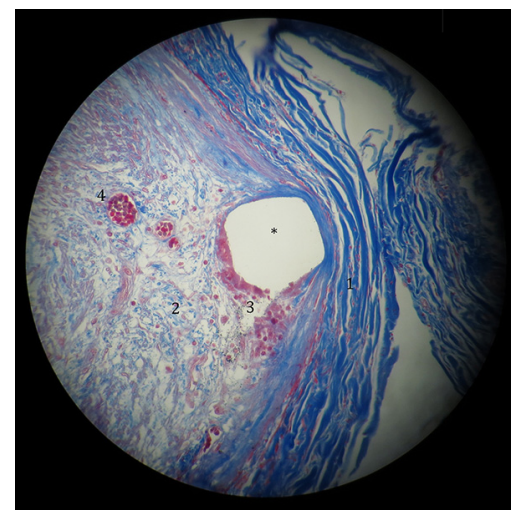

Figura 2 Fotomicrografía de arteria coronaria posterior a la electrodeposición que muestra el espacio que ocupaba el stent $\left({ }^{*}\right)$. Periféricamente hay gruesas fibras de colágena en color azul fuerte (1). Centralmente hay miofibroblastos (2) en una matriz de delgadas fibras de colágena (azul claro). Hay células gigantes multinucleadas (3) de reacción a cuerpo extraño en la periferia del stent (color rojo). Presencia de pequeños vasos de neoformación (4) (Masson $\times 40$ ).

\section{Responsabilidades éticas}

Protección de personas y animales. Los autores declaran que para esta investigación no se han realizado experimentos en seres humanos ni en animales.

Confidencialidad de los datos. Los autores declaran que en este artículo no aparecen datos de pacientes.

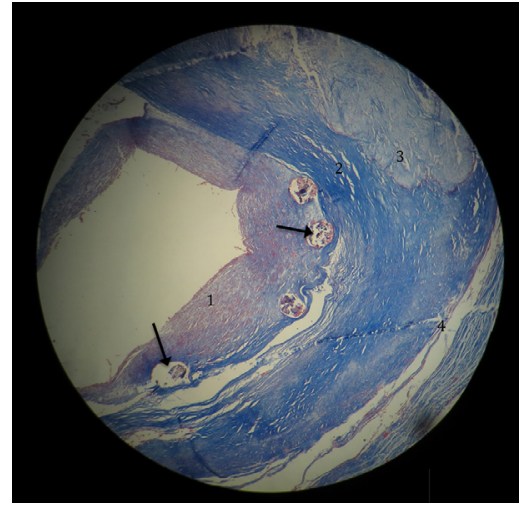

Figura 3 Fotomicrografía de pared arterial coronaria posterior a la electrodeposición, observe los espacios con remanentes de stent (flecha). Hacía la luz del vaso la proliferación de la neoíntima (1). Periféricamente se observa placa ateromatosa esclerótica (2) con área de necrosis (3). La capa muscular está atrófica y extremadamente adelgazada (4) (Masson $\times 2.5$ ).

Derecho a la privacidad y consentimiento informado. Los autores declaran que en este artículo no aparecen datos de pacientes.

\section{Financiación}

No se recibió patrocinio de ningún tipo para llevar a cabo este artículo.

\section{Conflicto de intereses}

Los autores declaran no tener ningún conflicto de intereses.

\section{Agradecimientos}

Al Dr. Biol. O. Infante, jefe del Departamento de Instrumentación Electromecánica, por facilitarnos el uso del laboratorio de electrónica, y al Dr. Abundes por facilitarnos diversos materiales para reproducir la técnica.

\section{Bibliografía}

1. Bradshaw SH, Kennedy L, Dexter DF, et al. A practical method to rapidly dissolve metallic stents. Cardiovasc Pathol. 2009;18.3:127-33.

2. Rippstein P, Black MK, Boivin M, et al. Comparison of processing and sectioning methodologies for arteries containing metallic stents. J Histochem Cytochem. 2006;54:673-81. 